\title{
Repair of incisional hernias - Experience with a combined fascial and prosthetic mesh repair.
}

\author{
S.M. Amjad Hossain ${ }^{1}$, Khairun Nahar ${ }^{2}$, J.I. Sen ${ }^{3}$
}

\begin{abstract}
Summary
Repairs of incisional (ventral) hernia is one of the commonly performed operation in Bangladesh. This is a prospective study conducted in Shaheed Suhrawardy Hospital, Dhaka and a private Hospital (BDM Hospital) at Dhaka city from June 2001 to 31st May 2004 with a total period of 3 years and with total patients 43 . Incisional hernias develop in upto $11 \%$ of surgical abdominal wounds with a possible recurrence, following repairs of $44 \%$. There are several methods of repair of incisional hernias, including laparoscopic method of repair which is gaining popularity day by day. But we describe our experience with a combined fascial and prosthetic mesh repair. Of total 43 patients treated, 27 were female \& 16 were male. The original operation was gynaecological in 27, bowel related surgery in 15 cases \& biliary surgery in 4 patients. The incisions were midline in 31 patients, transverse in 10 patients and paramedian in 2 patients. The hernias were considered subjectively to be large in 21, medium in 16 and small in 6 patients. A parameter was compiled for each patient, noting intraoperative and post postoperative complications, post operative hospital stay and analgesic requirements. Post operative complications included seroma formation in 6 patients. One patient developed wound infection and require removal of the mesh 10 Control infection. Post operative hospital stay ranged from 2 to 17 days. Of total 43 patients 36 were available for follow- up. Seven drops from follow up. Follow up was from 6 months to 36 months. One (2.5\%) of these patients complained of persistent lump and one reported persistent pain. Hernia recurrence in one patient (2.5\%) , 35 was found to have no recurrence. We advocate these technique because it is applicable to all hernias,most of the mesh is behind the rectus sheath and has two points of fixation, it is relatively pain free allowing early mobilization has a less complication rate and low recurrence rate.
\end{abstract}

Key Words: Incisional hernias, Prosthetic mesh.

\section{Introduction:}

Incisional hernias develop in 3.8-11.5\% patients after abdominal surgery ${ }^{1,2,3}$. The incidence depends on a number of factors including old age, male sex, obesity, bowel surgery, suture type, chest infection, abdominal distension and wound infection . Ninety percent of incisional hernias occur within 3 years of operation ${ }^{3}$.

Repair of large abdominal hernias is a difficult surgical problem with recurrence being a common problem. Recurrence rates of upto $33 \%$ after first repair and $44 \%$ after second repair have been reported.

1.Associate Professor (Surgery) Dhaka Medical College

2.Associate Surgeon (Gyane \& Obs.), Shaheed Suhrawardy Medical College \& Hospital, Dhaka.

3.Associate Surgeon Shaheed Suhrawardy Medical College \& Hospital, Dhaka.

\section{Correspondence:}

Associate Professor (Surgery) Dhaka Medical College
Numerous methods of repair have been described. Primary repair in one or two layers or Mayo-type overlap, use of fascia (local or naps) with suture derns and use of fascia with synthetic mesh (poly propylene mesh, marlex mesh, stainless steel or expanded polytetrafloroethyline) 1,4,3-16. In a literature review Loh et- al (1992) state that overlapping techniques produce impressive results and that techniques combining fascia with mesh have the advantage of overcoming excessive tension ${ }^{7,8,9}$.

We described our experience with a technique using fascia and polypropylene mesh, originally described by browse and honest (1979) ${ }^{10}$. This technique was initially used in the repair of long midline incisional hernias but subsequently was applied to subcostal hernias by whllty et al (1998) $)^{15}$. Our modification involves the introduction of an overlap and two points of anchor for the mesh.

\section{Patients and method:}

Operations were performed under general anaesthesia 
(24), spinal anaesthesia(15) and by epidural anaesthesia (4). After skin preparation and draping, the cutaneous scar was excised and the hernia sac dissected to expose the circumference of the abdominal wall defect (figure- 1). The sac was opened only if the sac was irreducible. The rectus sheath or external oblique aponeurosis was clearly exposed around the circumference of the defect. It was then incised at a distance from the edge of the defect, just to allow apposition of the lateral margin of the medial leafs after mobilization. The medial leaf was then elevated from the underlying muscle (figure-2) and its lateral margins sutured with continuous one zero prolene (polypropylene) investing the sac and in the case of midline hernias providing a midline fascial layers. In the case of transverse incisions care was taken to ensure that the circumferencial incision had aponeurotic or fibrous scar tissue on either side. In midline hernias the lateral leaf of the rectus sheath was then elevated from the underlying muscle. In transverse hernias the mesh was sutured to the under surface of the external oblique muscle with loose intcrrrupted prolene sutures.

The medial borders of the lateral leaf of the rectus sheath, or the fibrous margin of the lateral circumference of a transverse defect, was then sutured to the upper surface of the mesh with a continuous 2-0 prolene suture for u tension free repair.(ligure-S) . Skin closed with interrupted or subcuticular stitch by 2-0 prolene, after inserting two suction drains. The drains were removed when there was less than $50 \mathrm{ml}$ of drainage in 24 hours. The patients were mobilized post operatively, as soon as possible and discharged home once the drains had been removed.

Total 43 patients were evaluated of which 27 were female \& 16 were male. The original operation was gynaecological in 24 patients, bowel related surgery 15 patients \& biliary surgery in 4 patients. The original incision were midline in 31 patients, transverse in 10 patients \& paramedian in 2 patients. Five patients were recurrent incisional hernia, (had a single attempt at hernia repair), wound infection occurred in one patient and require removal of the mesh to control the infection. Follow up data was compiled from private chamber visit, Clinic visit and hospital visit in a proforma.

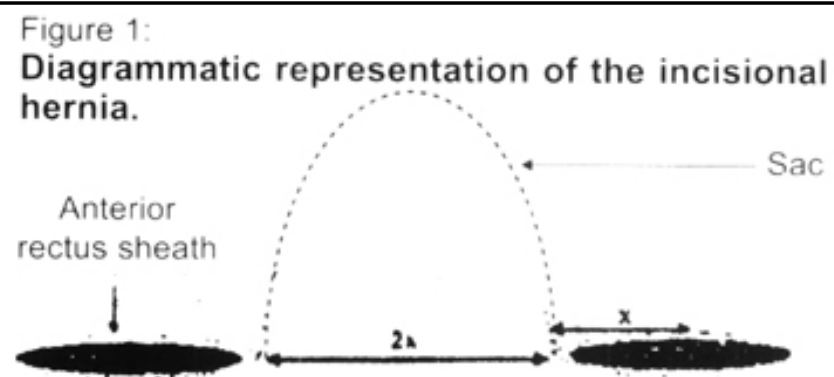

Figure 2:

The medial leaf of the divided rectus sheath is dissected free and reflected medially.

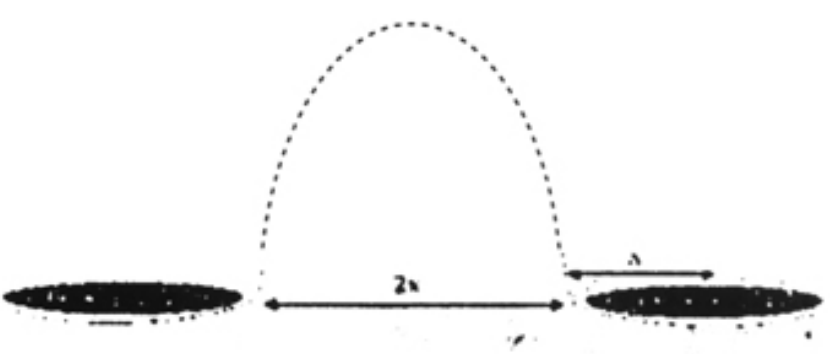

Figure 3:

The lateral leaf of the rectus sheath is sutured to the prolene mesh. The wound is then closed over suction drains.

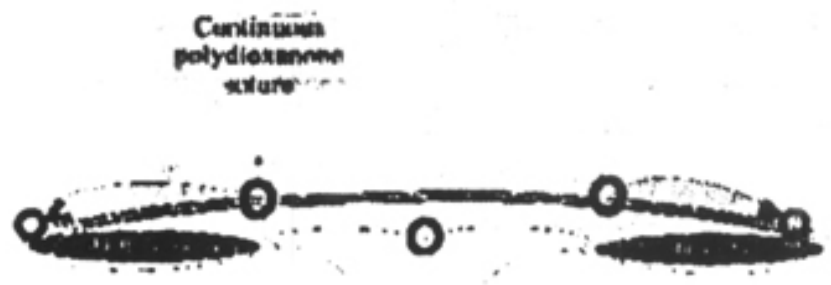

\section{Results:}

There were no intra operative complications. Seroma formation was the commonest problem with one patient requiring repeated aspirations upto 16 post operative day. Seroma formation of other 5 patients stops spontaneously within 8-10 days. One patient developed wound infection and after removal of the stitch wound dehiscence occur and require removal of the mesh to control infection. Post operative hospital stay ranged from 1-17 days with a mean of 5 days. Requirements of post operative analgesia according to the subjective size of the hernia.

In all patients wound healed without problems except one where wound infection occurred and required removal of the mesh. Of the 43 patients 36 wereavailable for follow up and 7 patients dropped from follow up. Follow up was from 6 months to 36 
Table-I:

Operation done, under anaesthesia

\begin{tabular}{|l|l|l|}
\hline Anaesthesia & Number & $\%$ \\
\hline General Anaesthesia & 24 & $58 \%$ \\
\hline Spinal Anaesthesia & 15 & $34 \%$ \\
\hline Epidural Anaesthesia & 4 & $9 \%$ \\
\hline
\end{tabular}

\section{Table-II:}

Post operative complications occurring in the 43 patients.

\begin{tabular}{|l|l|l|}
\hline Complications & Number & $\%$ \\
\hline Seroma formation & 6 & $14 \%$ \\
\hline Wound infection & 1 & $1.3 \%$ \\
\hline Urinary retention & 1 & $1.3 \%$ \\
\hline
\end{tabular}

months. One patient (2.5\%) complained of persistent lump and one (2.5\%) reported persistent pain Recurrence were found In one patient and remaining 35 patients were found to have no recurrence.

\section{Discussion:}

The use of u prosthetic mesh to repair large incisionul hernias is well established. Different techniques have been described including a "Sandwich" of mesh and rectus sheath with overlapping and two points of fixation, mesh placed deep to the rectus sheath with overlap and mattress suture fixation, a complex 'mesh peritonea sandwich', fixation of a large mesh anterior to the rectus sheath with two points of fixation and a combination of fascia and mesh 5'12 17. It has been suggested that overlapping leads to a better repair when one considers using fascia alone or in combination --with mesh s,13,14. x,18 Langer and Christiansen (1985) compared their results using primary repair with historical data using a mesh and suggested that the use of mesh gave a better repair with less recurrence. Loh et al (1992) in their literature review, suggested that the better results with mesh were simply a manifestation of inadequate follow up, besides they focused a number of complications associated with the use of a mesh ${ }^{9}$. Liakakos et al (1994) studied a prospective comparison.

Primary closure against the use of mesh and showed that the recurrence rate was less with mesh ${ }^{16}$.

Our study has incorporated a fasical repair with the mesh placed behind the anterior leaf of the rectus sheath with considerable overlap and two points of fixation. This method has been used for hernias arising from incisions other than those in the midline ${ }^{15}$.
Wound infection is a potentially major complication which is usually superficial but can be sever enough to necessitate removal of the mesh ${ }^{14}$. Mutapurkar et al (1991) reported no seroma formation because their mesh was incorporated into a peritoneal sandwich ${ }^{14}$. Formation of seroma was reported to be $4 \%$ by nu4lluy et al $(1991)^{17}$. Jacobs et al reported that seroma were easily managed by use of suction drains ${ }^{18}$.

We found one (2.5\%) recurrence at 11 months but it was due to infection and removal of the mesh. Previous studies have shown that $70-75 \%$ of recurrences within 2 years and $80-90 \%$ developed within the 3 years ${ }^{1,3,5}$

\section{Conclusion:}

We advocate this method of incisional hernia repair as it is applicable to all sites of incisional hernia, the mesh is mostly hidden behind the rectus sheath and is anchored with two points of fixation, there is relatively little pain allowing for early mobilization, the complication rate is low and there is a low recurrence rate.

\section{References:}

1. Langer S, Christiansen J. Long Term results after incisional hernia repair. Acta theirs Scand 1985" 151: 217.

2. Bucknell TE, C'ox PJ. Ellis 11. Burst abdomen and incisional hernia: a prospective study of 1129 major laparotomies. Br J 1982: 284:931-3.

3. Mudge M, Hughes LE. Incisional hernia: a 10 years prospective study of incidence and attitudes. Br J surg 1985: 72:70-1.

4. Young D. Repair of epigastric incisional hernia. Br J Surgery 1961: 48: 514-16.

5. Read RC. Yoder G. Recent trends in the management of incisional herniation. Arch Surg 1989:124:485-8.

6. Maguire J, Young D. Repair of epigastric incisional hernia $\mathrm{Br} \mathrm{J}$ surg 1976; 63:125-7.

7. Jenkins TPN. Incisional hernia repair: a mechanical approach. $\mathrm{Br} \mathrm{J}$ Surg 1980; 67: 335-6.

8. Van Der Linden FTPM. Van vroonhoven Tr JMV: Lung term results after surgical correction of incisional hernia. Netherlands Journal of Surgery 1988; 40:127-9.

9. Loh a, Rajkumar JS, South LM. Anatomical repair of large incisional hernias. Ann R Coll surg Engi 1992: 74: 100-5.

10. Browse NL, Hurst P. Repair of long, large midline incisional hernias using reflected flaps of anterior rectus sheath reinforced with mesh. Am J sug 1979:138:738-9.

11. Mannlner MJ, Lavonius M Perhoniemi VJ. Results of mcisiona hernia repair. A retrospective study of 172 unselected hernioplasties. Eur J Surg 1991: 157:29-31.

12. Vunter RR. Anatomical repair of midline incisional hernia $\mathrm{Br} \mathrm{J}$ Surg 1971: 58:888-91 Amzad.Khairun et al.

No.2, December 2009

13. Usher FC. New technique for repairing incisionai hernias with marlixmesh. Am J Surg 1979; 138:740-1.

14. Matapurkar BG. Gupta AK, Agarwal AK. A new technique of a "Marlex- Peritoneal Sandwich" in the repair of large incisionai hernias. World J surg 1991; 15: 768-70.

15. Whitley MS, Ray Chaudhuri SIS, Galland RB. Combined fascia and mesh closure of large incisionai hernias. Jr Coll Surg Edinb 1998:43:29-30.

16. Liakako T, Karanikas 1, Panagiotidis H, Dendrinos S. Use of marlex mesh in the repair of recurrent incisionai hernia. $\mathrm{Br} \mathrm{J}$ sung 
1994; 81: 248-9.

17. Molloy RG, Moran KT, Waldron RP, Brady MP, Kirwan WO. Massive incisionai hernia: abdominal wall replacement with marlex mesh. Br J Surg 1991; 78:242-4.
18. Jacobs E, Blais dell FW, Hall AD. Use of knitted marlex mesh in the repair of ventral hernias. Am J Surg 1965; 110: 897-902. 